\title{
P12: Entwicklung und Evaluierung eines Fragebogens zur Beurteilung von pathologischem Altruismus (FBPA)
}

\author{
Rosa Lori Teubner-Guerra $\cdot$ Elke Ochsmann
}

Online publiziert: 26. Oktober 2013

(C) Springer-Verlag Wien 2013

Einleitung/Fragestellung: Im Rahmen einer Entwicklungsstudie wurde pathologischer Altruismus mit dem selbst erstellten Fragebogen zur Beurteilung von pathologischem Altruismus (FBPA) erfasst und auf Zusammenhänge mit der ins Deutsche übersetzten amerikanischen Compassionate Altruism Scale (CAS) geprüft.

Mit dem FBPA sollen Faktoren für das Vorhandensein von pathologischem Altruismus ermittelt und die Items des FBPA reduziert werden. Mögliche Zusammenhänge zwischen Altruismus-Faktoren, Berufsgruppen und anderen soziodemographischen Einflussvariablen werden ermittelt und Interkorrelationen zwischen FBPA und CAS geprüft.

Methodik/Stichprobe: Der FBPA mit 76 Items wurde basierend auf Literaturrecherchen konstruiert und anhand einer Stichprobe $(n=259)$ überprüft. Mittels Faktorenanalysen wurden Faktoren für pathologischen Altruismus gewonnen. Die Reliabilität wurde geprüft. Varianzanalytisch wurden Zusammenhänge zwischen Altruismus-Faktoren und Berufsgruppen untersucht. Interkorrelationen zwischen dem FBPA und dem CAS wurden geprüft.
Ergebnisse: Die faktorielle Validität durch Faktorenextraktion erfolgte als Hauptkomponentenanalyse mit anschlieBender Varimax-Rotation und dem Reinheitskriterium nach Fürntratt. Hierdurch wurden 4 Faktoren mit den Eigenwerten $(8,8 ; 2,7 ; 2,3$ und 1,8$)$ ermittelt, die insgesamt $53,93 \%$ der Varianz erklärten. Die Reliabilität erwies sich mit Cronbach's Alpha $(\alpha=0,906$ bis $\alpha=0,709)$ als sehr zufriedenstellend. Die insgesamt 32 Items lassen sich als vier Skalen, die pathologischen Altruismus näher beschreiben, interpretieren und wurden umschrieben als ,pathologische, empathische Reaktion“ (Co-Abhängigkeit, massive Schuldgefühle), „übermäßiges Funktionieren“, „Selbstwertstabilisierung“ und ,idealisierte, erhöhte Wertvorstellungen“.

Es ergaben sich Zusammenhänge zwischen den Skalen des CAS und dem FBPA. Bestimmte Berufsgruppen zeigten höhere Werte für pathologischen Altruismus.

Diskussion/Schlussfolgerung: Mit dem FBPA-32 und dem CAS sollen unter anderem in der Hauptstudie Messinstrumente zur Diagnostik von pathologischem Altruismus standardisiert und normiert werden.

\footnotetext{
R. L. Teubner-Guerra

Praxis für Psychotherapie, Gera, Deutschland

E. Ochsmann

Westsächsische Hochschule Zwickau, Zwickau, Deutschland
} 